\title{
Paulownia tomentosa, como reservorio natural de gases antropogénicos en la región de Azuero
}

\section{Paulownia tomentosa, as a natural reservoir for anthropogenic gases in the Azuero region}

\author{
Ginger Carrasco ${ }^{l}$ Yuleinys Castro $^{l}$, José Norato $^{l}$, Anadalys Almengor ${ }^{l}$, Eny Serrano ${ }^{1 *}$ \\ ${ }^{1}$ Licenciatura en Ingeniería Civil, Centro Regional de Azuero, Universidad Tecnológica de Panamá
}

\begin{abstract}
Resumen La producción de $\mathrm{CO}_{2}$, tiene un impacto negativo en el ambiente. Lo que lleva a optar por soluciones que puedan contrarrestar la emisión de este gas de manera eficiente. Este proyecto presenta la reforestación con la especie Paulownia Tomentosa, para mitigar el impacto atmosférico producido por los vehículos a motor existentes en las ciudades de Chitré, Provincia de Herrera y La Villa de los Santos, Provincia de los Santos, las cuales están ubicadas en el Arco Seco de la República de Panamá. Teniendo en cuenta la capacidad de absorción de dióxido de carbono que tiene la especie, la cantidad de vehículos dentro de las localidades estudiadas y los reservorios naturales existentes en la zona, los datos apuntan a que la cantidad de árboles a sembrar de esta especie es menor a 2000 plantones.
\end{abstract}

Palabras clave Paulownia Tomentosa, dióxido de carbono, cambio climático, calentamiento global.

\begin{abstract}
Abstrac $\mathrm{CO}_{2}$ production has a negative impact on the environment, which leads to opting for solutions that can counteract the emission of this gas efficiently. This project presents the reforestation with the Paulownia Tomentosa species to mitigate the atmospheric impact produced by the existing motor vehicles in the cities of Chitré, Herrera Province and La Villa de los Santos, Los Santos Province. They are located in the Arco Seco (dry arch) of the Republic of Panama. Taking into account the carbon dioxide absorption capacity of the species, the number of vehicles within the places studied and the existing natural reservoirs in the area, the data indicates that the number of trees of this species to be planted is less than 2000 seedlings.
\end{abstract}

Keywords Paulownia Tomentosa, carbon dioxide, climate change, global warming.

\footnotetext{
* Corresponding author: eny.serrano@utp.ac.pa
}

\section{Introducción}

El calentamiento global es el aumento de la temperatura de la Tierra causada por las emisiones de $\mathrm{CO}_{2}$ y $\mathrm{CH}_{4}$ que realiza el efecto invernadero por acción del hombre [1]. Cada año en la Tierra se destruyen cerca de 13 millones de hectáreas forestales [2], por el mal uso del suelo, la deforestación y la quema de combustibles fósiles. Un ejemplo de este último es el petróleo, uno de los más utilizados a nivel mundial [3], productor de $\mathrm{CO}_{2}$, un gas contaminante.

La gasolina es un derivado del petróleo y la principal fuente de energía para los vehículos terrestres. Una consecuencia de su uso es la emisión diaria de $\mathrm{CO}_{2}$.

REDD+ (Reducción de emisiones por deforestación y degradación) es una iniciativa creada por la ONU-REDD para reducir las emisiones de $\mathrm{CO}_{2}$ [4] causadas por la deforestación y la degradación forestal en países en desarrollo, que busca promover la conservación y el manejo sostenible de los bosques, y aumentar las reservas de carbono. En América se llevan a cabo proyectos con la iniciativa de mitigar el cambio climático. Entre estos están: el proyecto Kiri Revolution ubicado en Texas (USA) [5] y "La Fábrica de Oxígeno" [6] en San Luis, Argentina, que utiliza al árbol para contrarrestar el $\mathrm{CO}_{2}$ en el ambiente y purificarlo.

El 70\% de las emisiones de $\mathrm{CO}_{2}$ (2016) [7] se generan en las ciudades. Panamá se ha visto afectada por esto; lo cual, ha provocado una reducción del $30 \%$ de las zonas boscosas y ocasionado un aumento de $\mathrm{CO}_{2}$ en el ambiente. Dejando como resultados ríos desérticos, y un aumento en la temperatura por la acumulación de gases de invernadero en la atmósfera. Esto trae como consecuencia ciudades y campos agrícolas destruidos, muchas zonas de desastres, inundaciones y sequías severas que causan múltiples daños.

Las emisiones de $\mathrm{CO}_{2}$ (2010) per cápita en Panamá son de 2.62 toneladas métricas [8] y una forma de contrarrestar esto sería plantar el árbol Paulownia Tomentosa, que en comparación con otras especies absorbe casi diez veces más $\mathrm{CO}_{2}$ [9] y se adapta a suelos pobres [10] aportándoles nitrógeno y oxígeno, lo cual contribuye a restablecer el ecosistema. 
Esta iniciativa se implementará en los distritos de Chitré y Los Santos, que en comparación con el resto del área boscosa del país presenta índices menores; por ende, se realizó un análisis de regresión matemática que consiste en utilizar datos proporcionados por el Instituto Nacional de Estadística y Censo y el Municipio de Panamá (Chitré y Los Santos) para estimar la cantidad de árboles de dicha especie que deberían ser plantados para restaurar el entorno.

\section{Antecedentes}

En Panamá se desarrollan múltiples proyectos en pro de la conservación del ambiente con un solo objetivo, recuperar el hábitat que poco a poco está desapareciendo.

Alianza por el millón de hectáreas es una iniciativa que tiene como objetivo reforestar un millón de hectáreas [11] en los próximos 20 años, cuyas componentes son la conservación de los bosques naturales, restauración de los bosques de galería y de las tierras degradadas.

Parte de esta iniciativa es el programa de inversión para la restauración de cuencas [12] hidrográficas prioritarias, Río Grande (Coclé), La Villa (Herrera, Los Santos) y Santa María (Veraguas, Ngäbe-Buglé, Herrera). Su objetivo general es restaurar y reforestar áreas de protección acuíferas y zonas degradadas en las cuencas hidrográficas priorizadas del país.

El proyecto "protección de reservas y sumideros de carbono en los manglares y áreas protegidas de Panamá" [13] busca demostrar la contribución que hacen los ecosistemas de manglar a la gestión del riesgo y de cambio climático. El proyecto contribuirá a la mitigación y adaptación al cambio climático y a la conservación de la biodiversidad.

La iniciativa REDD+ (Reducción de emisiones por deforestación y degradación) en Panamá se encarga de reducir las emisiones de dióxido de carbono causadas [14] por la deforestación y la degradación forestal en países en desarrollo, promover la conservación y el manejo sostenible de los bosques, y aumentar las reservas de carbono.

El Proyecto ecológico Azuero (PROECO), que desde 2010, se ha dedicado a la reforestación, desarrolla tres programas: Vivero de árboles, Huerto orgánico y Banco de semillas para la restauración de hábitat, manejo sostenible de tierras [15] y educación ambiental con la misión de conservar el ecosistema y proteger la biodiversidad.

\section{Metodología}

Para el desarrollo del proyecto fue necesario obtener los valores de la cantidad de autos que circulan en los distritos de Chitré y Los Santos, proporcionados por el Municipio de dichos distritos. Además, el total de autos que circulan en la República de Panamá.
Con estos valores se calcula el porcentaje de automóviles que circulan en ambos territorios (1)

$$
\%=\frac{\mathrm{AC}+\mathrm{AL}}{\mathrm{AT}} \mathrm{X} 100
$$

Dónde:

$\mathrm{AC}=$ Autos de Chitré

$\mathrm{AL}=$ Autos de Los Santos

AT $=$ Autos totales de la República de Panamá

Después, para obtener los valores necesarios, se totalizó la cantidad de combustible, es decir, gasolina y diésel (2) que se consume a nivel nacional.

$$
\mathrm{TC}=\mathrm{TD}+\mathrm{TG}
$$

Donde:

$\mathrm{TC}=$ Total de miles de galones de combustible

$\mathrm{TD}=$ Total de miles de galones de diésel

$\mathrm{TG}=$ Total de miles de galones de gasolina

Una vez obtenido esto, se multiplicó la cantidad total de combustible a nivel nacional por el porcentaje de autos de los distritos (3).

$$
\mathrm{GCL}=\mathrm{TC} \times \%
$$

Donde:

GCL $=$ Miles de galones de combustible en Chitré y Los Santos

Lo que dio como resultado los miles de galones que se consumen por año en las áreas de estudio. Luego se hizo la conversión de miles de galones a litros (4) para obtener los valores por año, mes, día, hora y minutos en el sistema internacional de medidas.

$$
\text { LPA }=\left(\frac{3.78541 \mathrm{~L}}{1 \text { Galón }}\right) \times \text { GCL }
$$

Por otro lado, para calcular las emisiones de $\mathrm{CO}_{2}$ que producen los autos, la calculadora huella de carbono para el transporte nos pide la distancia en kilómetros recorridos. Para obtener este valor, se evaluaron cuatro tipos de autos distintos, pequeño, mediano, grande y pick-up, que utilizan gasolina y diésel en una distancia conocida.

Estos datos se utilizan en conjunto con la cantidad de dinero con que se llenó cada auto, como resultado nos da la cantidad de litros que utilizan estos autos en la distancia ya conocida (5).

$$
\mathrm{LPD}=25\left(\frac{1 \mathrm{~L}}{\mathrm{~PB}}\right)
$$


Donde:

$\mathrm{CCB}=$ Cantidad de combustible comprada en balboas $\mathrm{PB}=$ Precio en Balboas

Luego, se multiplican los litros totales de combustible por los minutos antes obtenidos y por la distancia recorrida, todo esto entre la cantidad promedio de litros (6).

$$
\mathrm{DT}=\left(\frac{\text { LPM } x \text { TDR } x \text { DR }}{\text { LPDPromedio }}\right)
$$

Donde:

LPM= Litros totales por minuto

$\mathrm{TDR}=$ Tiempo total recorrida

$\mathrm{DR}=$ Distancia total recorrida

Este valor se introduce en la calculadora antes mencionada, para obtener el total de kilogramos de carbono que los autos de los distritos de Chitré y Los Santos producen.

Para conocer la cantidad de $\mathrm{CO}_{2}$ que es absorbida por los diferentes tipos de bosques en las áreas de estudio se multiplicó el promedio por año de consumo de $\mathrm{CO}_{2}$ por el total de árboles existentes, ambos por hectárea (7).

$$
\mathrm{CCO}=\mathrm{TCB} \times \mathrm{PA}
$$

Donde:

TCB $=$ Total de la cobertura boscosa por hectárea

PA = Promedio de absorción de los árboles en un año por hectárea

Como resultado nos da la cantidad de $\mathrm{CO}_{2}$ que es absorbida por año por los bosques.

Luego, restar la cantidad total de $\mathrm{CO}_{2}$ que absorben los árboles de la cantidad de $\mathrm{CO}_{2}$ que producen los autos por año (8).

$$
\Delta \mathrm{R}=\mathrm{LPA}-\mathrm{CCO}
$$

La diferencia de esto se divide entre la cantidad teórica promedia de kilogramos de $\mathrm{CO}_{2}$ que absorbe la Paulownia Tomentosa al año para conocer la cantidad promedio de este árbol a sembrar para mitigar $\mathrm{CO}_{2}$ restante.

$$
\text { Total de Paulownia Tomentosa }=\left(\frac{\Delta \mathrm{R}}{21.7 \mathrm{~kg}}\right)
$$

\section{Resultados}

Calculamos el porcentaje de autos que circulan en los distritos de Chitré y Los Santos con los datos suministrados por los Municipios y Contraloría General de la República de Panamá.

$$
\%=\frac{17516+6000}{783204} \times 100=3 \%
$$

Conociendo este porcentaje y TC, obtuvimos el consumo de miles de galones de combustible.

$$
\mathrm{GCL}=(310013+350718)(3 \%)
$$

GCL $=19821.93$ miles de galones de combustible

Con esto obtuvimos los litros que se consumen por año y minutos.

$$
\text { LPA }=\left(\frac{3.78541 \mathrm{~L}}{1 \text { Galón }}\right) \times 19821.93 \times 10^{3} \text { galones }
$$

$$
\text { LPA }=75.034132 \times 10^{6} \text { Litros por año }
$$

Litros por minutos $=144.741767$ Litros por minutos

Para el auto 1

$$
\mathrm{LPD}=25\left(\frac{1 \mathrm{~L}}{0.866}\right)=28.8684 \mathrm{~L}
$$

Para el auto 2

$$
\mathrm{LPD}=20\left(\frac{1 \mathrm{~L}}{0.845}\right)=23.6686 \mathrm{~L}
$$

Para el auto 3

$$
\mathrm{LPD}=30\left(\frac{1 \mathrm{~L}}{0.866}\right)=34.6420 \mathrm{~L}
$$

Para el auto 4

$$
\mathrm{LPD}=20\left(\frac{1 \mathrm{~L}}{0.758}\right)=26.3852 \mathrm{~L}
$$

Determinamos la distancia total en kilómetros que recorren todos los autos de Chitré y Los Santos, utilizando una distancia de $252.8 \mathrm{~km}$.

$$
\mathrm{DT}=\left(\frac{(144.741767 \mathrm{~L}) \times(195 \mathrm{~min}) \times(252.8 \mathrm{~km})}{28.391050 \mathrm{~L}}\right)
$$

$\mathrm{DT}=251313.2903 \mathrm{~km}$

El dióxido de carbono de que producen los autos de Chitré y Los Santos al año es de $128696262.5 \mathrm{~kg}$, lo que equivale a 1286962.625 toneladas, generado por la calculadora de huella de carbono para el transporte.

Calculamos la cantidad de $\mathrm{CO}_{2}$ que absorben los árboles en los distritos antes mencionados.

$$
\begin{gathered}
\mathrm{CCO}=(36963.34) \times(34.50 \text { toneladas })[16] \\
\mathrm{CCO}=1265235.23 \text { toneladas de } \mathrm{CO}_{2}
\end{gathered}
$$

Este valor nos permite conocer la cantidad promedio de Paulownia Tomentosa a sembrar. 


$$
\begin{gathered}
\Delta \mathrm{R}=1286962.625-1265253.23 \\
\Delta \mathrm{R}=11727.395 \text { toneladas de } \mathrm{CO}_{2} \\
\mathrm{TPT}=\left(\frac{32.1298 \text { toneladas }}{21.7 \mathrm{~kg}}\right)\left(\frac{1 \mathrm{~kg}}{0.001 \text { toneladas }}\right) \\
\text { Total de Paulownia Tomentosa }=1481 \text { árboles }
\end{gathered}
$$

\section{Conclusión}

En los distritos de Chitré y La Villa de Los Santos para el año 2017 se mantiene un registro vehicular hasta el desarrollo de este estudio de 23516, lo cuales emiten 1.27 x106 toneladas de $\mathrm{CO}_{2}$ por año aproximadamente.

$\mathrm{Al}$ obtener el promedio de $\mathrm{CO}_{2}$ que no es absorbido por los bosques existentes en los distritos de Chitré y Los Santos, podemos concluir que para mantener un reservorio de carbono óptimo entre ambos distritos, los cálculos matemáticos indican que con reforestar un aproximado de 1481 árboles de la especie Paulownia Tomentosa, podemos mitigar el impacto generado por los vehículos a motor de estas comunidades.

La Semilla de Paulownia Tomentosa es adaptable a suelos con un $\mathrm{pH}$ de entre $5.0-8.9$, lo que es favorable, ya que los suelos de la zona poseen características similares. En la provincia de Los Santos el pH oscila entre 5.6 - 5.9, lo que corresponde a un $36 \%$ de sus suelos y en la provincia de Herrera es de 5.0 correspondiente a un 51\%. Esto nos muestra una alta probabilidad de que este árbol germine y podamos verlo como una opción para solucionar esta problemática [15].

\section{REFERENCIAS}

[1] A. Says, (12/10/2018), "Calentamiento Global”. [Online]Available: http://queesela.net/calentamiento-global/

[2] ONU P. Thompson, (1/05/2017). [Online]. Available: www.news.un.org/es/story/2017/05/1378001

[3] C. Pérez Morales, (28/8/16) "Posibles Consecuencias del Cambio Climático". [Online].

Available:https://www.panamaamerica.com.pa/opinion/posiblesconsecuencias-del-cambio-climatico-1040433
[4] Mi Ambiente, (07/02/2018). [Online]. Available:http://www.miambiente.gob.pa/redd/index.php?option =com_content\&view=article\&id=140:para-que-se-creoredd $\&$ catid $=84 \&$ Itemid $=580 \&$ lang $=$ es

[5] K. Okura, Ch. Sanders, K. Suigura, (25/03/2013) "Kiri Revolution". [Online]. Available: https://www.thekiritree.com/history

[6] Marcelo Cantó, (07/06/2017). [Online] Available: http://argentear.com/kiri/

[7] Yanelis Dominguez, (14/01/2018). [Online]. Available: https://www.diaadia.com.pa/el-pais/panama en-lalucha-contra-la-huella-de-carbono-y-cambio-climatico-331958

[8] Secretaría Nacional de Energía, (2017) [Online].Available: http://www.energia.gob.pa/

[9] La Vanguardia. (04/01/2017) [Online]. "Este es el árbol que puede ayudar a combater el cambio climático". Available: www.lavanguardia.com/natural/20170102/413034036992/arbolkiri-cambio-climatico

[10] M. Marimar, (27-02-2018), "La Revolución del Kiri, el árbol que puede salvar el Mundo. [Online]

Available: www.elblogverde.com/la-revolucion-del- kiri-elarbol-que-puede-salvar-al-mundo/

[11] Mi Ambiente, “Alianza por El Millón”, [Online] Available:https://www.alianzaporelmillon.org/?page_id=366

[12] Mi Ambiente, "Alianza por El Millón", [Online].Available: https://www.alianzaporelmillon.org/?page_id=7005

[13] $\mathrm{Mi}$ Ambiente, "REDD+", [Online].Available: http://www.miambiente.gob.pa/redd/index.php?lang=es

[14] Proyecto Ecológico Azuero, (PROECO), [Online].Available: https://proecoazuero.org/quienes-somos/

[15] J. Villarreal, I. Ramos, J. Villalaz, A. Santo, (abril 2016). Caracterización de los suelos de la región de Azuero-Panamá para elaboración de mapas detallados, Instituto de Investigación Agropecuaria de Panamá. [Online]. Available: https://www.researchgate.net/publication/301291324

[16] Dr. M. G. Manzano, Ing. J.C. Hernández, (15/05/08). Estimación de la Captura y Almacenamiento de Carbonic en Ecosistemas de la Reserva de la Biósfera Sierra Luna. [Online] Available:http://www.katoombagroup.org/ katoomba/docume nts/events/event19/Captura_y_almacen_de_carbono_MManza no.pdf 